\title{
Determination of mechanical properties of okra, sisal fibre and polyester based hybrid composite
}

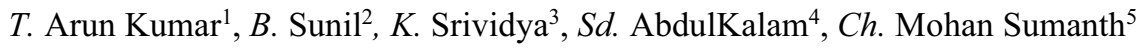 \\ ${ }^{1,2}$ IV B.TechStudent, Prasad V Potluri Siddhartha Institute of Technology, Kanuru, Vijayawada, India \\ ${ }^{3}$ Associate Professor, Prasad V Potluri Siddhartha Institute of Technology, Kanuru, Vijayawada, India \\ ${ }^{4,5}$ Assistant Professor, Prasad V Potluri Siddhartha Institute of Technology, Kanuru, Vijayawada, India
}

\begin{abstract}
The natural fibre reinforced composites have the advantage of being light weight, renewable, cheap and eco-friendly when compared to the synthetic fibres. So, there is a need to investigate potentiality of natural fibre which can be used in highly demanding situations. An attempt has been made in the present work to explore the possible uses of variety of cultivated and wild grown fibres in nature that aids in the development of new composites for load carrying structures. The present research work has been carried out to make use of okra /sisal natural fibres. The aim of this paper is to describe the experimental development and characterization of new set of hybrid natural fibre composites which is made by reinforcing okra /sisal fibres with polyester resin in matrix by using hand layup method. The natural fibres were extracted by retting and combing process manually. The hybrid composites were prepared using okra/sisal fibres of $30 / 70,50 / 50,70 / 30$ fibre weight ratios, when subjected to varying weights of fibres $(0.4,0.8,1.2,1.6,2)$ grams. The tensileand impact properties were carried out using hybrid composite specimens.
\end{abstract}

\section{Introduction}

\subsection{Composites:}

A material is considered as a compositematerial when it has two or more distinct constituent materials which are bound together. A composite material includes fibre and matrix which are held together, and properties like strength and stiffness can be increased by it. Fibres act as the load carrying member while matrix aids in proper orientation and it is used as a load transfer medium.Composites are mainly classified as Fibrous, Laminated and Particulate Composites.Resins are widely used as matrix materials.Particulate composites are produced by suspending particles of one or more materials in a matrix of another material.

\subsection{Fibres:}

A fibre looks like a thread or filament which can be obtained from leaves, stems and roots from fibre yielding plants. A fibre is classified into two types. i.e., Natural and Synthetic fibres. Natural fibres are obtained from natural resources where Synthetic fibres are manmade like polyester, rayon, nylon etc. In order to meet the industrial applications natural fibre-reinforced composites are selected as they are renewable and cheap. These are used in many applications like automobile, aerospace and military applications. The fibres that are used in the present experiment are Okra and Sisal fibres.

\subsection{Matrices:}

The material used in a matrix is polymer resin which can get hardened by leaving it in an open air for a certain period of time. In addition to maintaining the shape of the composite structure, aligning the reinforcements, and acting as a stress transfer medium, the matrix also protects the fibres from abrasion and corrosion. The two basic classes of resins are thermo sets and thermoplastics which differ by their unique behaviour when they get heated. Thermo sets undergo an irreversible chemical change when they are cured while thermoplastics act as reversible as they melt when heated and gets solidified when cooled.

\subsection{Vegetable Fibres:}

Since a long time, mankind has been depending on plants for Textile fibres. For instance, cotton was cultivated in India even before 1600's. Almost any plant fibre is made up of two materials which are cellulose and lignin. There are 6 kinds of fibres that can be classified under vegetable fibres which are textile, brush, planting, filling, natural and paper making fibres. 1)Textile Fibres: There are three subclassifications under it which are surface fibres, soft fibres and hard fibres. Cotton comes

\footnotetext{
* Corresponding author: srividya.kode@gmail.com
} 
under surface fibres, whereas Jute and Kenaf come under soft fibres. Species of Agave fibres such as Maguey, Mexican, Juamave etc. come under Hard fibres. 2)Brush fibres: The kind of fibre that is used for making brushes and mats are Leaf, leaf stalk and inflorescence stalk of Palmyra, and Kittul. 3)Planting and rough weaving fibres: Applications such as hats, mats, baskets, etc. employ fibres extracted from palm trees and grasses. 4)Filling fibres: fibres of red silk, mandar, white silk cotton, cat tail plants are used in making of stuffing pillows, cushions, etc. 5) NaturalFibre: Fibres of Upas trees, Paper trees, tauri, Cuba bast and lacebark are some of alternatives for clothing fibres but they come with some drawbacks. 6) Paper making fibres: In paper industry, woods of several plants, such as cotton, linen, bamboo, paper mulberry, bagasse and many grasses and straw are used.

[1] N.Srinivasababu et.al have fabricated composites using okra, sisal and banana fibres and concluded that the tensile strength of the material gets enhanced by using chemical extraction. [2] Sanjay M R et.al have made have made investigations on mechanical properties evaluated from jute glass fibre reinforced plastic and found that the tensile strength and impact strength results were good when the composition of the jute and glass fibre are equal in proportion.[3]M. T. Isa et.al have investigated on the effects of fibre treatment and found that it greatly improves the properties of natural fibres that creates a rough surface for good adhesion.[4] N.Srinivasababu et.al explained on how okra fibre is to be extracted and the optimum time required to keep the okra stems in mud water.[5] C.Chaithanyan et.al evaluated the mechanical properties of Coir-Sisal Reinforced Hybrid Composites Using Isophthalic Polyester Resin and concluded that the breaking load of sisal-glass fibre is higher than sisal-coir-glass and coirglass fibres.[6] Arpitha G R et.al have conducted tests on the epoxy based hybrid composites reinforced with Sisal, SIC, Glass fibres and concluded that sisal, glass fibre composition yields good strengths.[7] V.Surendra et.al have fabricated composites using sisal, jute and okra fibre and found that the results were good and jute combination gives more strength.[8] Nadella srinivasababu gave an overview of okra fibre reinforced polymer composite and concluded that it was quite useful.

\section{Collection of extracted fibres:}

\subsection{Extraction of Sisal Fibre:}

Sisal fibre is extracted from leaves of the Agave plant by using Decortication process. The leaves of the sisal plant originate at top of the stem and are dark green in colour with waxy surface. To extract the fibre the leaves are cut down and are made to pass through the rollers, and theleaves get crushed during the passage. The crushed leaves are washed using water and then made to dry naturally or by using mechanical source resulting in which the fibres can be used for experimentation.

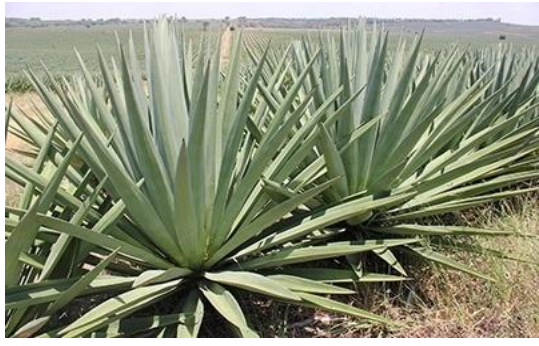

Figure1.Sisal plant

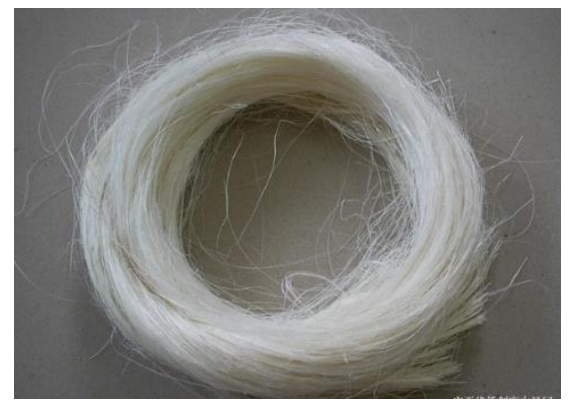

Figure 2. Sisal fibre

\subsection{Extraction of Okra Fibre:}

The fibre is collected from the okra plant from its stem wastes which are left out after the harvest. To extract the fibre from the plant the okra stems are cut down and were placed in a mud water pit which is in standstill condition. The stems were left out in the pit for 6 days and on the next day the stems are to be washed thoroughly with water such that the pulp gets detached from the fibre. As the pulp gets detached, it is left out with fibreand this fibre can be used by drying it for a week under the sunlight.

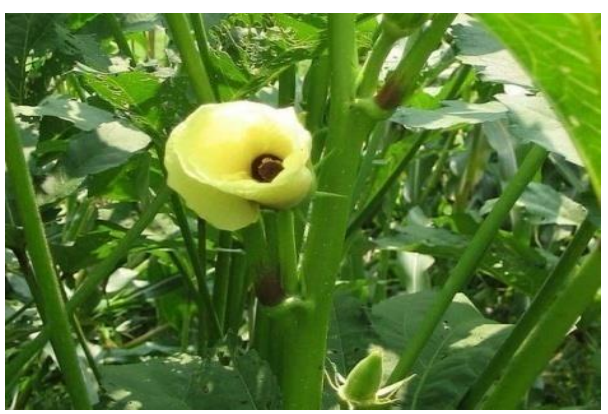

Figure 3. Okra plant

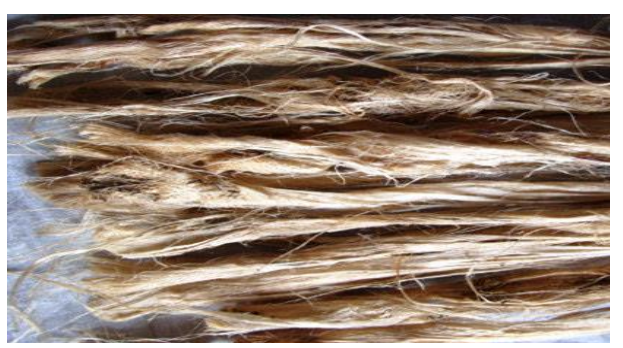

Figure 4. Okra fibre 


\section{Fabrication details:}

ASTM-D790M-86 is selected to get the dimensions of the test specimens. The mould is prepared on smooth ceramic tile whose surface is cleaned with a thinner, with rubber shoe sole to the required dimension. Mansion hygienic wax is applied on the tile surface to ensure that the specimen does not get sticked to the tile and to fill the gap between the rubber sole and the tile and it is left over until the wax gets dried. The mould is filled with polyester resin using the hand layup method. ECMALON 4411which is an unsaturated polyester resin is used in the present experimentation. Cobalt accelerator and methyl ethyl ketone peroxide (MEKP) catalyst are used which undergoes chemical reaction to cure the resin. In order to avoid explosion during stirring catalyst is to be added at last after the resin and accelerator gets mixed.The resin is poured in the mould in the form of thin layer and then fibres of measured weight are placed on the resin layer. Then the rest of the mould is filled with resin making sure that that there are no air gaps. After the specimens get hardened, they are filed and are tested using testing equipment.The okra, sisal fibres are selected in the ratios of 30/70,50/50,70/30 okra/sisal fibre composites with fibre weights $(0.4,0.8,1.2,1.6$, and 2 grams). The weight fraction of fibre present in the specimen is determined for each set.

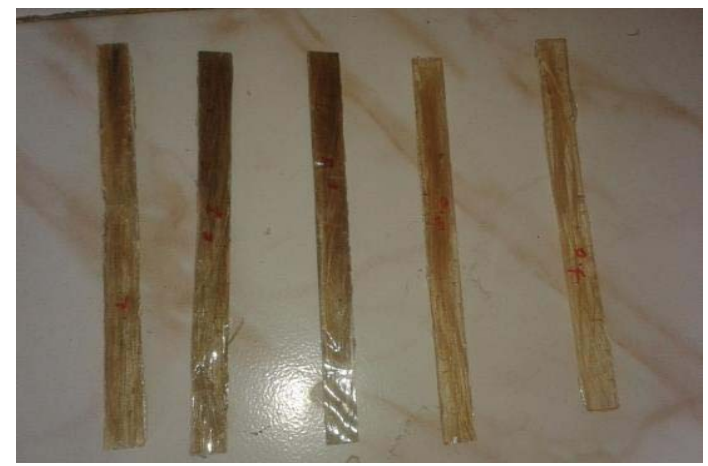

Figure 5. Specimens prepare for tensile test

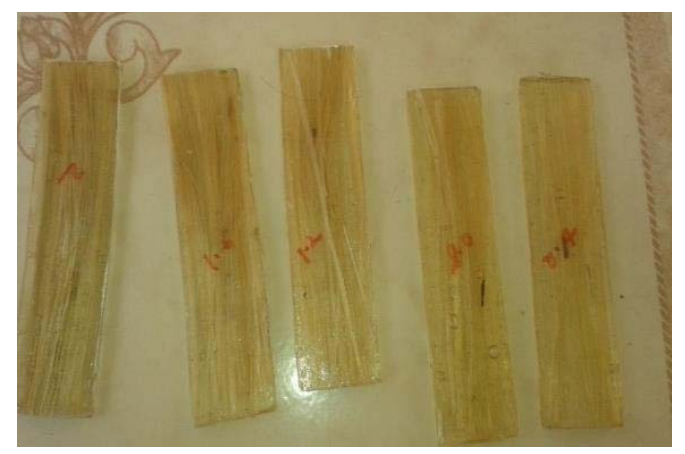

Figure 6. Specimens prepared for testing flexural strength

After the specimens have got cured, they are tested for results. Each specimen is marked in order to avoid confusion and swapping of the values. Standard testing equipment and methods are used to test the tensile strength, flexural strength and impact strength of the specimens.

\section{Results and discussions:}

The specimens after undergoing tests the tensile strength, flexural strength and impact strength are found.

\subsection{Tensile Strength Analysis:}

Table1: Mean ultimate tensile strength with respect to volume fraction of Hybrid okra-sisal (30/70) composite.

\begin{tabular}{|c|c|c|c|}
\hline S.NO. & $\begin{array}{c}\text { Weight of } \\
\text { the fibre } \\
\text { (g) }\end{array}$ & $\begin{array}{c}\text { Volume } \\
\text { fraction } \\
\text { (Vf) }\end{array}$ & $\begin{array}{c}\text { Tensile } \\
\text { strength } \\
\text { (MPa) }\end{array}$ \\
\hline 1 & 0.4 & 14.32 & 37.06 \\
\hline 2 & 0.8 & 18.67 & 45.41 \\
\hline 3 & 1.2 & 22.16 & 56 \\
\hline 4 & 1.6 & 26.74 & 65.6 \\
\hline 5 & 2 & 29.68 & 73.3 \\
\hline
\end{tabular}

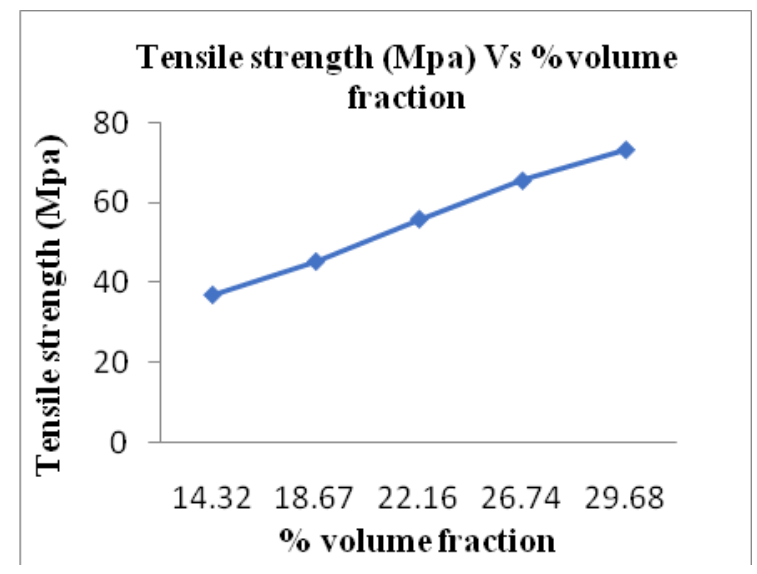

Graph 1. Tensile strength Vs volume fraction of hybrid okrasisal (30/70) fibre composite

By analysing the graph, it is clear that tensile strength is increasing with increasing the weight of fibre. The tensile strength of hybrid (okra-sisal) fibre at volume fraction 29.68 is $73.3 \mathrm{MPa}$. 
Table2.Mean ultimate tensile strength with respect to volume fraction of Hybrid okra-sisal (50/50) composite.

\begin{tabular}{|c|c|c|c|}
\hline S.NO. & $\begin{array}{l}\text { Weight of the } \\
\text { fibre }(\mathbf{g})\end{array}$ & $\begin{array}{l}\text { Volume } \\
\text { fraction } \\
\left(\mathbf{V}_{\mathbf{f}}\right)\end{array}$ & $\begin{array}{l}\text { Tensile } \\
\text { strength } \\
(\mathbf{M P a})\end{array}$ \\
\hline 1 & 0.4 & 13.2 & 38.93 \\
\hline 2 & 0.8 & 17.9 & 48.08 \\
\hline 3 & 1.2 & 21.07 & 65.06 \\
\hline 4 & 1.6 & 25.13 & 72.08 \\
\hline 5 & 2 & 29.03 & 86.6 \\
\hline
\end{tabular}

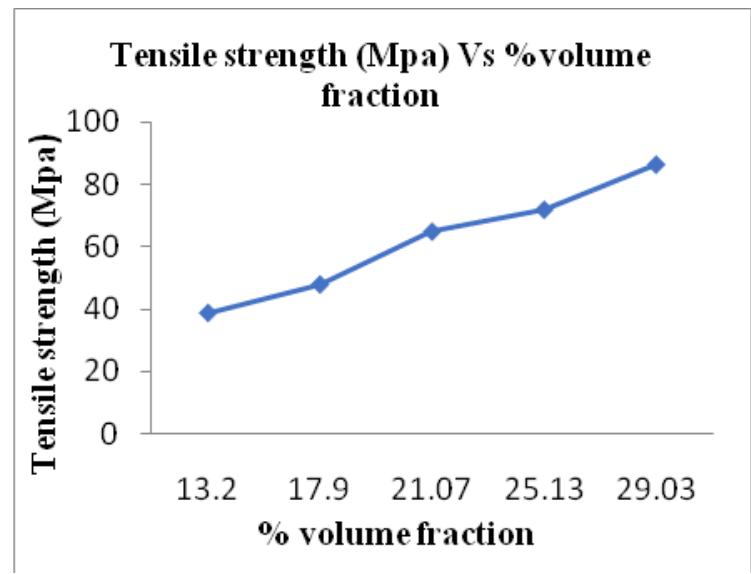

Graph 2. Tensile strength Vs volume fraction of hybrid okrasisal (50/50) fibre composite.

It can be observed from the graph that the tensile strength of hybrid (okra-sisal) fibre at volume fraction 29.03 is $86.6 \mathrm{MPa}$. By analysing the graph, it is clear that tensile strength is increasing with increasing the weight of fibre

Table3: Mean ultimate tensile strength with respect to volume fraction of hybrid okra-sisal (70/30) composite.

\begin{tabular}{|c|c|c|c|}
\hline S.NO & $\begin{array}{l}\text { Weight of } \\
\text { the fibre (g) }\end{array}$ & $\begin{array}{l}\text { Volume } \\
\text { Fraction } \\
\left(\mathbf{V}_{\mathbf{f}}\right.\end{array}$ & $\begin{array}{l}\text { Tensile } \\
\text { strength } \\
(\mathbf{M P a})\end{array}$ \\
\hline 1 & 0.4 & 12.8 & 53.06 \\
\hline 2 & 0.8 & 16.7 & 63.46 \\
\hline 3 & 1.2 & 20.88 & 78.4 \\
\hline 4 & 1.6 & 24.9 & 87.7 \\
\hline 5 & 2 & 28.56 & 95.2 \\
\hline
\end{tabular}

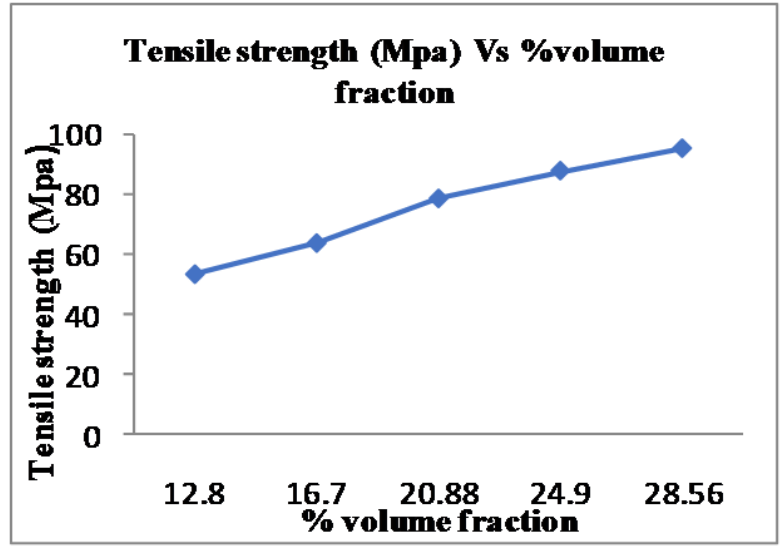

Graph 3. Tensile strength Vs volume fraction of hybrid okrasisal (70/30) fibre composite.

It can be observed from the graph that the tensile strength of hybrid (okra-sisal) fibre at volume fraction 28.56 is $95.2 \mathrm{MPa}$. By analysing the graph, it is clear that tensile strength is increasing with increasing the weight of fibre.

\subsection{Impact Strength Analysis:}

Impact strength is defined as the ratio between the energy observed by the specimen to the thickness of the composite.

Table 4. Mean ultimate impact strength with respect to volume fraction of hybrid okra-sisal (30/70) composite.

\begin{tabular}{|c|c|c|c|}
\hline S.NO & $\begin{array}{c}\text { Weight of the } \\
\text { fibre (g) }\end{array}$ & $\begin{array}{c}\text { Volume } \\
\text { Fraction } \\
\text { (Vf) }\end{array}$ & $\begin{array}{c}\text { Impact } \\
\text { strength } \\
\text { (J/M) }\end{array}$ \\
\hline 1 & 0.4 & 17.2 & 87.3 \\
\hline 2 & 0.8 & 23.6 & 127.73 \\
\hline 3 & 1.2 & 28.78 & 210 \\
\hline 4 & 1.6 & 33.12 & 273.6 \\
\hline 5 & 2 & 36.81 & 328.64 \\
\hline
\end{tabular}

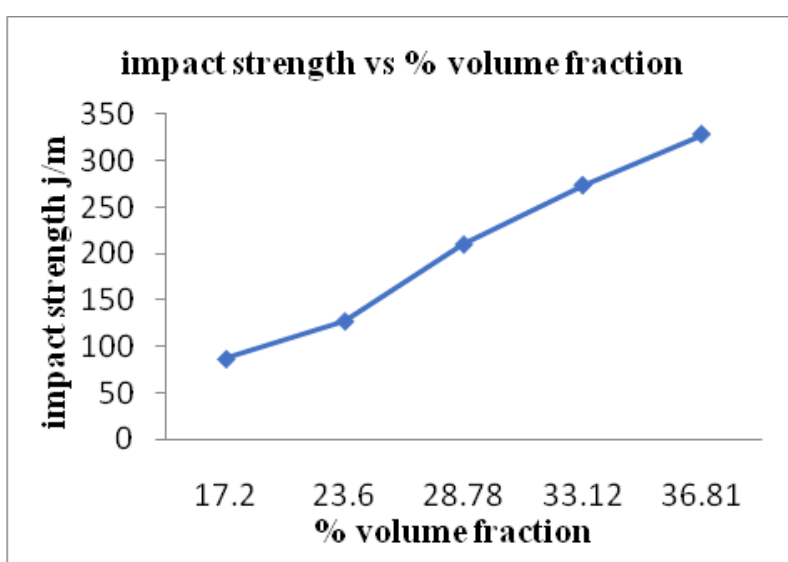

Graph 4. Impact strength Vs volume fraction of hybrid okrasisal (30/70) fibre composite

By analysing the graph, it is clear that impact strength is increasing with increasing the weight of fibre.Impact strength of hybrid (okra-sisal) fibre at volume fraction 36.81 is $328.16 \mathrm{j} / \mathrm{m}$. 
Table 5. Mean ultimate impact strength with respect to volume fraction of hybrid okra-sisal (50/50) composite.

\begin{tabular}{|c|c|c|c|}
\hline S.NO & $\begin{array}{c}\text { Weight of } \\
\text { the fiber } \\
(\mathbf{g})\end{array}$ & $\begin{array}{c}\text { Volume } \\
\text { Fraction } \\
\left(\mathbf{V}_{\mathbf{f}}\right)\end{array}$ & $\begin{array}{c}\text { Impact } \\
\text { strength } \mathbf{( J / M )}\end{array}$ \\
\hline 1 & 0.4 & 16.9 & 101.36 \\
\hline 2 & 0.8 & 22.82 & 135.42 \\
\hline 3 & 1.2 & 26.82 & 219.21 \\
\hline 4 & 1.6 & 33.75 & 303.72 \\
\hline 5 & 2 & 34.15 & 371.87 \\
\hline
\end{tabular}

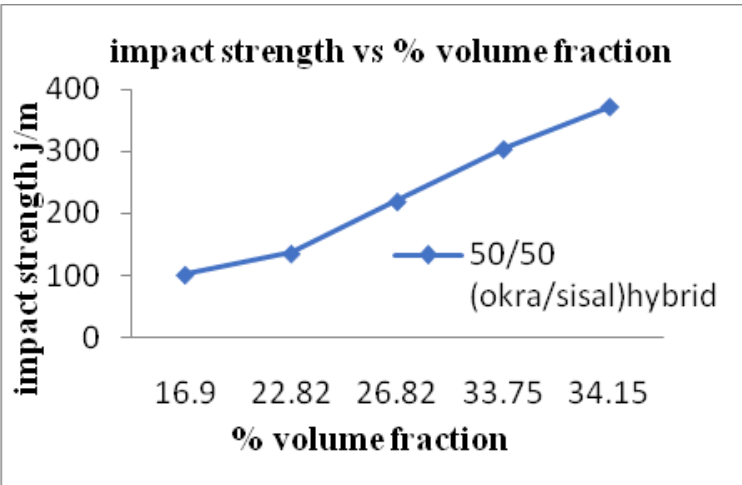

Graph 5. Impact strength Vs volume fraction of hybrid okrasisal (50/50) fibre composite

By analysing the graph, it is clear that impact strength gets increased by increasing the weight of fibre. Impact strength of hybrid (okra-sisal) fibre at volume fraction 34.15 is $371.87 \mathrm{j} / \mathrm{m}$.

Table 6. Mean ultimate impact strength with respect to volume fraction of hybrid okra-sisal (70/30) composite.

\begin{tabular}{|c|c|c|c|}
\hline S.NO & $\begin{array}{c}\text { Weight of } \\
\text { the fibre (g) }\end{array}$ & $\begin{array}{c}\text { Volume } \\
\text { Fraction } \\
\left(\mathbf{V}_{\mathbf{f}}\right)\end{array}$ & $\begin{array}{c}\text { Impact } \\
\text { strength } \\
(\mathbf{J} / \mathbf{M})\end{array}$ \\
\hline 1 & 0.4 & 15.19 & 92.6 \\
\hline 2 & 0.8 & 20.1 & 130.72 \\
\hline 3 & 1.2 & 24.36 & 211.12 \\
\hline 4 & 1.6 & 29.7 & 286.23 \\
\hline 5 & 2 & 32.32 & 323.72 \\
\hline
\end{tabular}

By analysing the values, it is clear that impact strength gets increased by increasing the weight of fibre. Impact strength of hybrid (okra-sisal) fibre at volume fraction 32.32 is $323.72 \mathrm{j} / \mathrm{m}$.

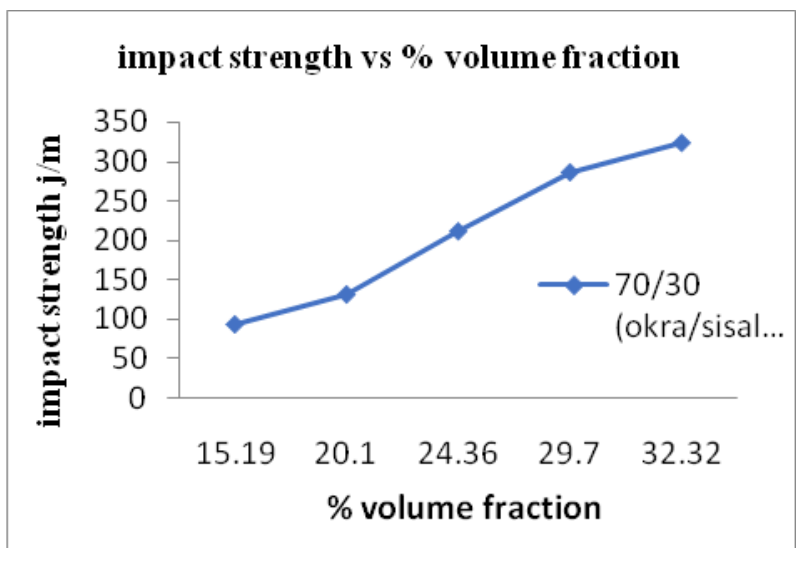

Graph 6. Impact strength Vs volume fraction of hybrid okrasisal (70/30) fibre composite.

\subsection{Flexural Strength Analysis:}

Maximum flexural stress(S)can be found out using the given relation.

$$
S=\frac{3 P L}{2 b t^{3}}(1)
$$

Where maximum load is represented by $\mathrm{P}(\mathrm{N})$. Span length, width and thickness of specimen are represented by $1, b, t$ in $\mathrm{mm}$ respectively.

Table 7.Mean ultimate flexural strength with respect to volume fraction of Hybrid okra-sisal (30/70) composite.

\begin{tabular}{|c|c|c|c|}
\hline S.NO & $\begin{array}{c}\text { Weight of } \\
\text { the fibre } \\
\text { (g) }\end{array}$ & $\begin{array}{c}\text { Volume } \\
\text { Fraction } \\
\text { (Vf) }\end{array}$ & $\begin{array}{c}\text { Flexural } \\
\text { Strength } \\
\text { (MPa) }\end{array}$ \\
\hline 1 & 0.4 & 11.06 & 74.6 \\
\hline 2 & 0.8 & 15.7 & 84 \\
\hline 3 & 1.2 & 23.62 & 105 \\
\hline 4 & 1.6 & 27.89 & 119 \\
\hline 5 & 2 & 32.67 & 135.5 \\
\hline
\end{tabular}

By analysing the graph, it is clear that flexural strength is increasing with increasing the weight of fibre. The flexural strength of hybrid (okra-sisal) fibre at volume fraction 32.67 is $135.5 \mathrm{MPa}$.

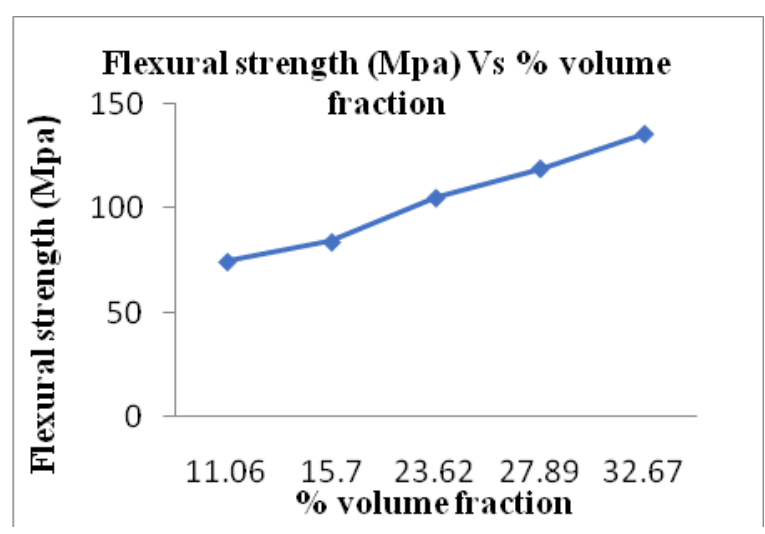

Graph 7. Flexural strength Vs volume fraction of hybrid okrasisal (30/70) fibre composite. 
Table 8. Mean ultimate flexural modulus with respect to volume fraction of Hybrid okra-sisal (50/50) composite.

\begin{tabular}{|c|c|c|c|}
\hline S.NO & $\begin{array}{c}\text { Weight of } \\
\text { the fibre } \\
(\mathbf{g})\end{array}$ & $\begin{array}{c}\text { Volume } \\
\text { Fraction } \\
(\mathbf{V f})\end{array}$ & $\begin{array}{c}\text { Flexural } \\
\text { Strength } \\
\text { (MPa) }\end{array}$ \\
\hline 1 & 0.4 & 10.7 & 84 \\
\hline 2 & 0.8 & 17.03 & 93.3 \\
\hline 3 & 1.2 & 22.73 & 109.6 \\
\hline 4 & 1.6 & 26.32 & 130.6 \\
\hline 5 & 2 & 31.5 & 158.6 \\
\hline
\end{tabular}

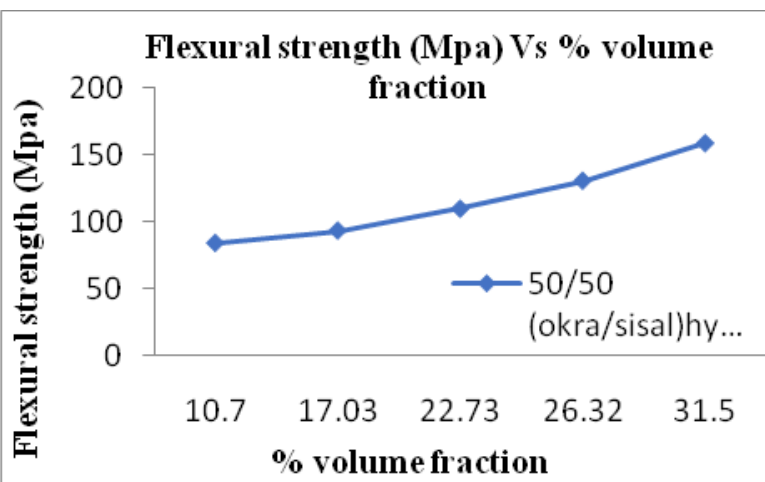

Graph 8.Flexural strength Vs volume fraction of hybrid okrasisal (50/50) fibre composite

By analysing the graph, it is clear that flexural strength is increasing with increasing the weight of fibre. The flexural strength of hybrid (okra-sisal) fibre at volume fraction 31.5 is $158.6 \mathrm{MPa}$.

Table 9.Mean ultimate flexural strength with respect to volume fraction of Hybrid okra-sisal (70/30) composite.

\begin{tabular}{|c|c|c|c|}
\hline S.NO & $\begin{array}{c}\text { Weight of } \\
\text { the fibre } \\
\text { (g) }\end{array}$ & $\begin{array}{c}\text { Volume } \\
\text { Fraction } \\
\text { (Vf) }\end{array}$ & $\begin{array}{c}\text { Flexural } \\
\text { strength } \\
\text { (MPa) }\end{array}$ \\
\hline 1 & 0.4 & 10.32 & 86.3 \\
\hline 2 & 0.8 & 16.88 & 119 \\
\hline 3 & 1.2 & 23.8 & 140 \\
\hline 4 & 1.6 & 27.82 & 158.06 \\
\hline 5 & 2 & 31.06 & 182 \\
\hline
\end{tabular}

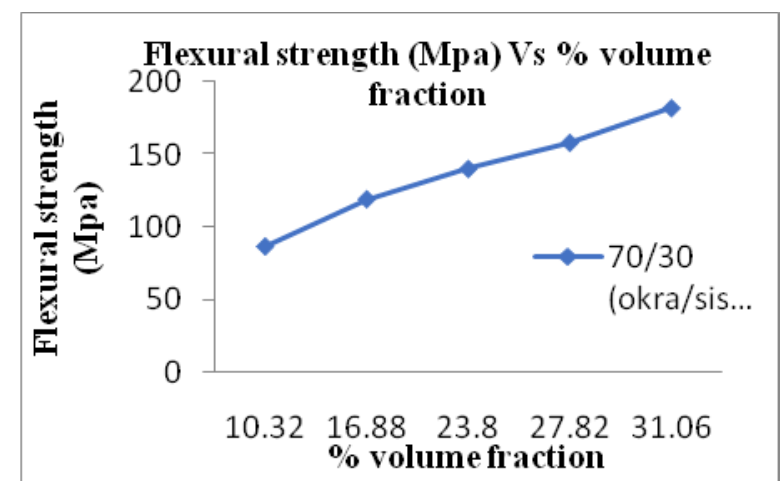

Graph 9.Flexural strength Vs volume fraction of hybrid okrasisal (70/30) fibre composite

By analysing the graph, it is clear that flexural strength is increasing with increasing the weight of fibre. The flexural strength of hybrid (okra-sisal) fibre at volume fraction 31.06 is $182 \mathrm{MPa}$.

\section{Conclusion:}

The sisal-okra natural fibre reinforced composite samples are fabricated by the hand layup method. Tests have been conducted on the specimens at different volume fractions to find out mechanical properties such as tensile and impact strength, and from the results obtained the following conclusions are drawn.

- The hybrid composite (okra/sisal) samples are exhibiting better impact strength.

- The flexural strength of the composite increases as the percentage of okra fibre in the composition increases. This indicates that okra fibre aids in withstanding more flexural loads.

- Since the weight of the okra fibre is less, the quantity of fibre is more at all weight ratios compared to the sisal fibre.

- It can be observed that tensile strength and impact strength gets increased by increasing the weight of the fibre.

- The hybrid okra-sisal composite can be used as an alternative material for many applications as they exhibit good strength.

\section{References:}

[1] Srinivasababu N, Rao KM, Kumar JS. Indian Journal of Science and Technology; 2, 35 (2009).

[2] Sanjay M R, Arpitha G R, B Yogesha. IOSR Journal of Mechanical and Civil Engineering (IOSR-JMCE) 11, 50 (2014).

[3] M. T. Isa, S. Usman, A. o. Ameh, O.A. Ajayi, O. Omorogbe, S. U. Ameuru. International Journal of Materials Engineering, 4, 180 (2014).

[4]N Srinivasababu, K.Murali Mohan Rao, J Suresh kumar. International Journal of Engineering, 3, 403 (2009).

[5]C.Chaithanyan, H.Venkatasubramanian, Dr. S.Raghuraman,T. Panneerselvam. International 
Journal of Innovative Research in Science,Engineering and Technology, 2 (2013).

[6] Arpitha G R, Sanjay M R, L Laxmana Naik, B Yogesha. International Journal of Engineering Research and General Science, 2 (2014).

[7] Surendra IV, Rao KV, Chandu KV. International Journal of Engineering Trends and Technology (IJETT); 19, 116 (2015).

[8] Srinivasababu N. In IOP conference series: materials science and engineering, 83, 012003 (2015). 